\title{
Mixed parity pairing in a dipolar gas
}

\author{
G. M. Bruun ${ }^{1}$ and C. Hainzl and M. Laux ${ }^{2}$ \\ ${ }^{1}$ Department of Physics and Astronomy, University of Aarhus, Ny Munkegade, DK-8000 Aarhus C, Denmark \\ ${ }^{2}$ Mathematisches Institut, Universität Tübingen, Auf der Morgenstelle 10, 72076 Tübingen, Germany
}

(Dated: October 17, 2018)

\begin{abstract}
We show that fermionic dipoles in a two-layer geometry form Cooper pairs with both singlet and triplet components, when they are tilted with respect to the normal of the planes. The mixed parity pairing arises because the interaction between dipoles in the two different layers is not inversion symmetric. We use an efficient eigenvalue approach to calculate the zero temperature phase diagram of the system as a function of the dipole orientation and the layer distance. The phase diagram contains purely triplet as well as mixed singlet and triplet superfluid phases. We show in detail how the pair wave function for dipoles residing in different layers smoothly changes from singlet to triplet symmetry as the orientation of the dipoles is changed. Our results indicate that dipolar quantum gases can be used to unambiguously observe mixed parity pairing.
\end{abstract}

\section{INTRODUCTION}

Time-reversal symmetry and inversion symmetry play a key role in the formation of Cooper pairs. Anderson showed that time-reversal symmetry ensures the existence of degenerate states for $s$-wave singlet Cooper pairing [1], whereas triplet pairing relies on inversion symmetry [2]. The breaking of these symmetries has profound effects on pairing. Superconductivity in crystals with no inversion symmetry is intensely studied, since it can lead to Cooper pairs with both spin-singlet and spintriplet components and therefore no definite parity $[3,4]$. While there are many crystals lacking inversion symmetry, only few experiments indicate mixed parity pairing. Evidence for mixed parity pairing has been reported for $\mathrm{Li}_{2} \mathrm{Pt}_{3} \mathrm{~B}[5,6]$, and measurements on $\mathrm{CePt}_{3} \mathrm{Si}$ crystals are consistent with mixed parity pairing [7, 8], but can also be attributed to multiband and disorder effects [9].

In this article, we show that dipolar fermions residing in two parallel layers can form a superfluid characterised by mixed parity pairing. The dipoles are aligned by an external field, and when their dipole moment is tilted with respect to the normal of the plane, the interaction between dipoles residing in the two different layers is not inversion symmetric, which leads to mixed parity pairing with both singlet and triplet components. Using a computationally efficient eigenvalue approach, we investigate the competition between the mixed parity interlayer pairing and the pairing between dipoles in the same layers, which is in the triplet channel. We map out the resulting zero temperature phase diagram as a function of the dipole tilting angle and the layer distance. Finally, we demonstrate in detail how the interlayer pairing gradually changes from being purely in the singlet channel when the dipoles are perpendicular to the layers, to being purely in the triplet channel when they are parallel to the layers.

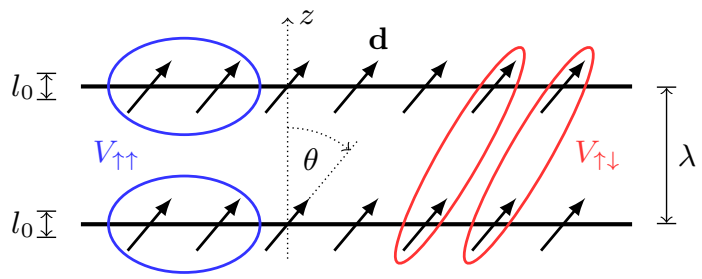

FIG. 1. Dipoles are moving in two layers separated by the distance $\lambda$. An external field aligns the dipoles so that they form an angle $\theta$ with respect to the normal of the planes. Intralayer/interlayer Cooper pairs driven by the interaction $V_{\uparrow \uparrow} / V_{\uparrow \downarrow}$ are indicated by blue and red ellipses respectively.

\section{SYSTEM}

We consider dipolar fermions of mass $m$ which reside in two parallel layers separated by a distance $\lambda$. The dipoles are confined in each layer by a strong harmonic potential perpendicular to the layer ( $z$-direction), which "freezes" the dipoles in the lowest harmonic oscillator state in the $z$-direction $\phi\left(z-z_{s}\right)=\exp \left[-\left(z-z_{s}\right)^{2} / 2 l_{0}^{2}\right] \pi^{-1 / 4} l_{0}^{-1 / 2}$, where $z_{s}$ is the position of layer $s$ along the $z$-direction and $l_{0}$ is the layer thickness. This confinement makes the system effectively two-dimensional (2D). The density of dipoles is $n=k_{F}^{2} / 4 \pi$ in each layer where $k_{F}$ is the Fermi momentum, and we denote dipoles in the upper layer as (pseudo) spin $\uparrow$ and dipoles in the lower layer as spin $\downarrow$. The dipoles are aligned by an external field so that their dipole moment $\mathbf{d}$ lies in the $x z$-plane forming an angle $\theta$ with the normal to the layers. We illustrate the setup in Fig. 1. The dipole-dipole interaction is $V(\mathbf{r})=$ $D^{2}\left(1-3 \cos ^{2} \theta_{r d}\right) / r^{3}$, where $\theta_{r d}$ is the angle between $\mathbf{d}$ and the relative displacement vector $\mathbf{r}$ of the two dipoles. We have $D^{2}=d^{2} / 4 \pi \epsilon_{0}$ for electric dipoles and $D^{2}=$ $d^{2} \mu_{0} / 4 \pi$ for magnetic ones. 


\section{A. Effective 2D Hamiltonian}

The effective interaction between dipoles in layer $s$ and $s^{\prime}$ is obtained by integrating the dipole interaction $V(\mathbf{r})$ over the Gaussians centered in each layer: $V_{s s^{\prime}}\left(\mathbf{r}_{\perp}-\mathbf{r}_{\perp}^{\prime}\right)=$ $\int d z \int d z^{\prime} \phi\left(z-z_{s}\right) V\left(\mathbf{r}-\mathbf{r}^{\prime}\right) \phi\left(z^{\prime}-z_{s^{\prime}}\right)$, where $\mathbf{r}_{\perp}=(x, y)$ is the $2 \mathrm{D}$ position of a dipole in a layer. Importantly, we see that the interlayer interaction is not inversion symmetric, i.e. $V_{\uparrow \downarrow}\left(-\mathbf{r}_{\perp}\right) \neq V_{\uparrow \downarrow}\left(\mathbf{r}_{\perp}\right)$ for $0<\theta<\pi / 2$. Instead, it obeys the symmetry $V_{\uparrow \downarrow}\left(-\mathbf{r}_{\perp}\right)=V_{\downarrow \uparrow}\left(\mathbf{r}_{\perp}\right)$ where the two layers are interchanged, and inversion symmetry is recovered only for $\theta=0$ and $\theta=\pi / 2$. On the other hand, the intralayer interaction is always inversion symmetric with $V_{\downarrow \downarrow}\left(\mathbf{r}_{\perp}\right)=V_{\uparrow \uparrow}\left(\mathbf{r}_{\perp}\right)=V_{\uparrow \uparrow}\left(-\mathbf{r}_{\perp}\right)$. Performing a $2 \mathrm{D}$ Fourier transform yields [10]

$$
V_{\uparrow \uparrow}(\mathbf{q})=-2 \pi D^{2} F\left(l_{0} q\right)\left[\cos ^{2} \theta-\sin ^{2} \theta \cos ^{2} \phi\right]
$$

for the intralayer interaction, where $F(x)=$ $q \exp \left(x^{2} / 2\right) \operatorname{erfc}(\mathrm{x} / \sqrt{2})$ and $\mathbf{q}=\left(q_{x}, q_{y}\right)$. Here, $\phi$ is the azimuthal angle of $\mathbf{q}$, and we have ignored a constant term (depending on $\theta$ ) in Eq. (1), since it plays no role for identical fermions. The interlayer interaction is [11]

$$
V_{\uparrow \downarrow}(\mathbf{q})=2 \pi D^{2}(i \cos \theta-\sin \theta \cos \phi)^{2} q e^{-\lambda q}
$$

for $l_{0} \ll \lambda$. We have $V_{\uparrow \downarrow}(-\mathbf{k})=V_{\uparrow \downarrow}(\mathbf{k})^{*}$.

These interactions give the effective 2D Hamiltonian for the bi-layer system

$$
\begin{gathered}
H=\sum_{\mathbf{k}, s} \frac{k^{2}}{2 m} c_{\mathbf{k} s}^{\dagger} c_{\mathbf{k} s}+\frac{1}{\mathcal{V}} \sum_{\mathbf{k}, \mathbf{k}^{\prime}, \mathbf{q}} V_{\uparrow \downarrow}(\mathbf{q}) c_{\mathbf{k}+\mathbf{q} \uparrow}^{\dagger} c_{\mathbf{k}^{\prime}-\mathbf{q} \downarrow}^{\dagger} c_{\mathbf{k}^{\prime} \downarrow} c_{\mathbf{k} \uparrow} \\
+\frac{1}{2 \mathcal{V}} \sum_{s} \sum_{\mathbf{k}, \mathbf{k}^{\prime}, \mathbf{q}} V_{\uparrow \uparrow}(\mathbf{q}) c_{\mathbf{k}+\mathbf{q} s}^{\dagger} c_{\mathbf{k}^{\prime}-\mathbf{q} s}^{\dagger} c_{\mathbf{k}^{\prime} s} c_{\mathbf{k} s}
\end{gathered}
$$

where we have used $V_{\uparrow \downarrow}(-\mathbf{q})=V_{\downarrow \uparrow}(\mathbf{q})$. Here, $\mathcal{V}$ is the volume of the system, and $c_{\mathbf{k} s}$ removes a dipole in layer $s=\uparrow, \downarrow$ with 2D momentum $\mathbf{k}$.

\section{PAIRING}

The intra- and interlayer interaction has attractive regions, and we will now examine the resulting Cooper pairing between dipoles residing in the same layer (intralayer pairing) as well as pairing between dipoles residing in the two different layers (interlayer pairing).

\section{A. Interlayer pairing}

The interaction between dipoles in the two different layers has attractive regions for all dipole angles $\theta$. For the special case of the dipoles perpendicular to planes $(\theta=0)$, the interlayer pairing has been examined using BCS theory without $[12,13]$ and with induced interactions [14], with variational methods [15], as well as with Monte-Carlo methods [16]. Here, we investigate the nature of the interlayer pairing for a general $\theta$. To do this, we use BCS theory introducing the anomalous average $\left\langle c_{-\mathbf{k} \downarrow} c_{\mathbf{k} \uparrow}\right\rangle$, which describes interlayer pairing for a translationally invariant system. Defining $\Delta(\mathbf{k})=\mathcal{V}^{-1} \sum_{\mathbf{k}^{\prime}} V_{\uparrow \downarrow}\left(\mathbf{k}-\mathbf{k}^{\prime}\right)\left\langle a_{-\mathbf{k}^{\prime} \downarrow} a_{\mathbf{k}^{\prime} \uparrow}\right\rangle$, the gap equation becomes

$$
\Delta(\mathbf{k})=-\frac{1}{\mathcal{V}} \sum_{\mathbf{k}^{\prime}} V_{\uparrow \downarrow}\left(\mathbf{k}-\mathbf{k}^{\prime}\right) \frac{\Delta\left(\mathbf{k}^{\prime}\right)}{2 E_{\mathbf{k}^{\prime}}}
$$

where $E_{\mathbf{k}}=\sqrt{\xi_{k}^{2}+|\Delta(\mathbf{k})|^{2}}$ and $\xi_{\mathbf{k}}=k^{2} / 2 m-\mu$ with $\mu$ the chemical potential. Due to Fermi statistics, we can write the anomalous average as

$$
\left\langle a_{-\mathbf{k} s^{\prime}} a_{\mathbf{k} s}\right\rangle=A \phi_{t}(\mathbf{k}) \cdot \sigma_{x}+B \phi_{s}(\mathbf{k}) \cdot \sigma_{y}
$$

where $\sigma_{x}=\left(\begin{array}{ll}0 & 1 \\ 1 & 0\end{array}\right)$ and $\sigma_{y}=\left(\begin{array}{cc}0 & -i \\ i & 0\end{array}\right)$ are the Pauli matrices, and $A$ and $B$ are constants. The coefficient $A$ corresponds to interlayer triplet pairing with $\phi_{t}(\mathbf{k})=$ $-\phi_{t}(-\mathbf{k})$, and $B$ corresponds to interlayer singlet pairing with $\phi_{s}(\mathbf{k})=\phi_{s}(-\mathbf{k})$. Since $V_{\uparrow \downarrow}(\mathbf{k}) \neq V_{\uparrow \downarrow}(-\mathbf{k})$ for $0<$ $\theta<\pi / 2$, it follows from Eq. (4) that $\Delta(\mathbf{k}) \neq \pm \Delta(-\mathbf{k})$. Thus, a pure singlet or triplet pair wave function is not a solution to Eq. (4), and the interlayer pairing has both $A$ and $B$ different from zero corresponding to a mixed parity. Note that this mixed parity pairing is due to an interaction without inversion symmetry, in contrast to the case of non-centrosymmetric metals where the possibility of mixed parity pairing arises due to a lack of inversion symmetry of the underlying crystal lattice [17].

\section{B. Intralayer pairing}

The intra-layer interaction between two dipoles residing in the same layer has been shown to give rise to pairing beyond a critical tilting angle $\theta_{c} \simeq \arcsin (2 / 3) \simeq$ $0.23 \pi[18]$. We include this pairing instability by the anomalous averages $\left\langle c_{-\mathbf{k} \uparrow} c_{\mathbf{k} \uparrow}\right\rangle$ and $\left\langle c_{-\mathbf{k} \downarrow} c_{\mathbf{k} \downarrow}\right\rangle$. The intralayer pairing is in the triplet channel, and for a single layer, numerical calculations show that it is predominantly of $p$-wave character with a small $f$-wave component for some angles $\theta[18-20]$. The gap equation for intralayer pairing is obtained from Eq. (4) simply by replacing $V_{\uparrow \downarrow}$ with $V_{\uparrow \uparrow}$.

\section{Eigenvalue analysis}

We will investigate the competition between inter- and intralayer pairing and in particular, what the ground state of the system is for different tilting angles $\theta$ and layer distances $\lambda$. To do this, we could solve in principle solve the corresponding non-linear gap equations taking a pairing field with a general symmetry. There is fortunately a much simpler and more accurate approach. A considerable effort has in recent years been put into the 
mathematical analysis [21-24] of the gap-equation (4) for rather general pair interactions $V\left(\mathbf{k}-\mathbf{k}^{\prime}\right)$, in the thermodynamic limit $\mathcal{V} \rightarrow \infty$. For a comprehensive review see [25]. Among other things it was rigorously proven that, in the weak coupling limit, the critical temperature [22, 23], as well as the gap [23] $\Delta\left(\mathbf{k}_{F}\right)$ on the Fermi surface, depends only on the lowest eigenvalue of an appropriate operator acting on functions which are living on the Fermi-surface. There is only pairing if this eigenvalue is negative. For a circular Fermi-surface in 2D this operator has the specific form

$$
A_{V} u(\varphi)=\frac{1}{4 \pi} \int_{0}^{2 \pi} V\left(\varphi, \varphi^{\prime}\right) u\left(\varphi^{\prime}\right) \mathrm{d} \varphi^{\prime},
$$

where $V\left(\varphi, \varphi^{\prime}\right) \equiv V\left(k_{F}\left(\cos \varphi-\cos \varphi^{\prime}\right), k_{F}\left(\sin \varphi-\sin \varphi^{\prime}\right)\right)$ is the interaction potential between momenta at the Fermi surface with azimuthal angles $\varphi$ and $\varphi^{\prime}$. The lowest eigenvalue $e(V)$ of the operator $A_{V}$ plays the role of the scattering length in the sense that $[22,23]$

$$
T_{c}(V) \simeq \frac{8 e^{\gamma-2}}{\pi} e^{\frac{1}{k_{F} e(V)}} .
$$

Since the operator $A_{V}$ given by Eq. (6) is linear and acts on functions living on the Fermi surface only, it is numerically much easier to analyse, compared to solving the full non-linear gap equation in the whole of $\mathbf{k}$-space. Indeed, our approach based on Eq. (6) allows us to obtain accurate results in the weak coupling limit for both intraand interlayer pairing without too much numerical effort. The numerical method is described in App. A.

\section{PHASE DIAGRAM}

We now use this eigenvalue approach to examine the ground state of the system. For all numerical calculations, we use the layer thickness $k_{F} l_{0}=0.11$. Since the relative strength of the intrashell and intershell pairing is independent of the dipole moment $D$, the nature of the ground state depends only on the two dimensionless parameters $\theta$ and $k_{F} \lambda$. For given values of $\theta$ and $k_{F} \lambda$, the ground state is determined by the lowest negative eigenvalue of $A_{V}$ : If $e\left(V_{\uparrow \downarrow}\right)<e\left(V_{\uparrow \uparrow}\right)$ the ground state is a superfluid with interlayer pairing, whereas the ground state is an intralayer superfluid if $e\left(V_{\uparrow \uparrow}\right)<e\left(V_{\uparrow \downarrow}\right)$. Note that $e\left(V_{\uparrow \downarrow}\right)<0$ for all $\theta$. The possibility of simultaneous interlayer and intralayer pairing is not considered here, since the presence of one order parameter typically suppresses other kinds of order by gapping the Fermi surface.

In Fig. 2, we plot the resulting $T=0$ phase diagram. For $\theta<\theta_{c} \simeq 0.23 \pi$, there is no intralayer pairing and the ground state is therefore a superfluid with interlayer pairing for all layer separations $k_{F} \lambda$. Of course, the critical temperature of the superfluid is vanishingly small for large separations, since the interlayer interaction becomes very small. In the limit of large layer separation $k_{F} \lambda \gg 1$, the intralayer pairing therefore wins as soon as

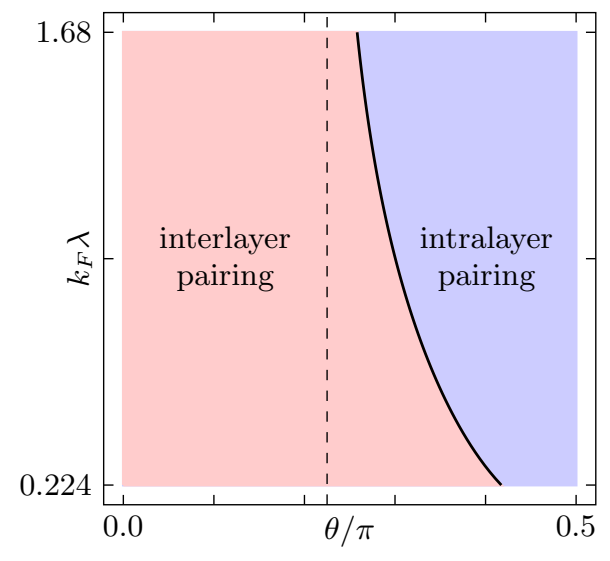

FIG. 2. The ground state of the bi-layer system as a function of $\theta$ and $k_{F} \lambda$. For $\theta \leq \theta_{c} \simeq 0.23 \pi$ (vertical dashed line), the interlayer pairing always dominates since $A_{V_{\uparrow \uparrow}}$ has no negative eigenvalue.

$\theta>\theta_{c}$ as is indicated by the vertical dashed line in Fig. 2. When the layer distance decreases and the strength of the interlayer interaction increases, Fig. 2 shows how the interlayer superfluid is the ground state for increasingly large angles $\theta$ beyond $\theta_{c}$. We have not plotted the phase diagram for very small $k_{F} \lambda$ since the condition $l_{0} \ll \lambda$ for using Eq. (2) breaks down in this regime.

To illustrate how the phase diagram is obtained, we plot in Fig. 3 the lowest eigenvalues $e\left(V_{\uparrow \downarrow}\right)$ and $e\left(V_{\uparrow \uparrow}\right)$ corresponding to interlayer and intralayer pairing respectively, as a function of $k_{F} \lambda$ for $\theta=\pi / 3$, and as a function of $\theta$ for $k_{F} \lambda=1$. The system exhibits a quantum phase transition between inter- and intralayer pairing when the eigenvalues cross. Note that it is crucial for obtaining $e\left(V_{\uparrow \uparrow}\right)$ that only eigenvalues corresponding to antisymmetric eigenfunctions are allowed.

\section{SYMMETRY OF THE INTERLAYER PAIRING}

We now discuss in more detail the symmetry of the interlayer pairing. Figure 4 shows the interlayer pair wave function $\alpha(\varphi)$, which is the eigenfunction of $A_{V_{\uparrow \downarrow}}$ with the lowest eigenvalue, as a function of the azimuthal angle $\varphi$ on the Fermi surface, for three different tilting angles: $\theta=0, \theta=\pi / 4$, and $\theta=\pi / 2$. The layer distance is $k_{F} \lambda=1$. As expected, the pair wave function is purely singlet $s$-wave for $\theta=0$ reflecting that the interlayer interaction is rotationally symmetric when the dipoles are perpendicular to the layers. When $\theta=\pi / 4$ on the other hand, we see that the pair wave function has no definite parity with $\alpha(\varphi+\pi) \neq \pm \alpha(\varphi)$ corresponding to both $A \neq 0$ and $B \neq 0$ in Eq. (5). The pairing is purely in the triplet channel with $\alpha(\varphi+\pi)=-\alpha(\varphi)$ for $\theta=\pi / 2$, where the interlayer is inversion symmetric.

To quantify the symmetry of the pair wave function further, we split it into a symmetric and an anti- 

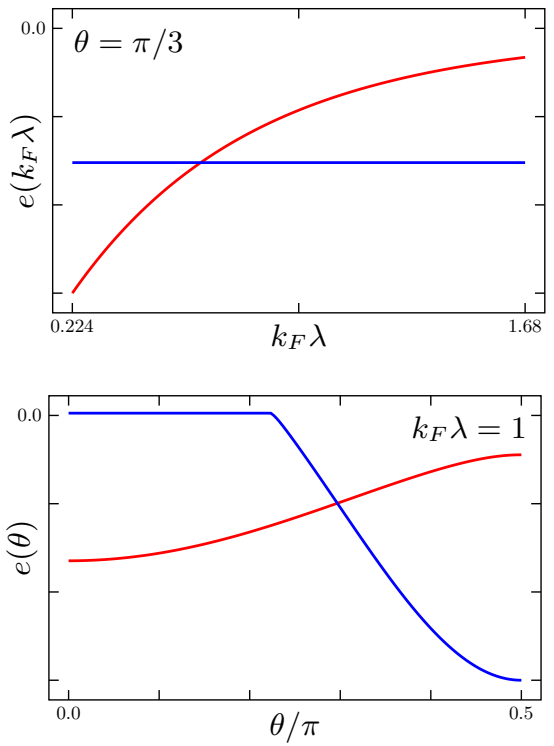

FIG. 3. Lowest eigenvalues $e\left(V_{\uparrow \downarrow}\right)$ (red) and $e\left(V_{\uparrow \uparrow}\right)$ (blue) as a function of the layer distance and the tilting angle respectively.
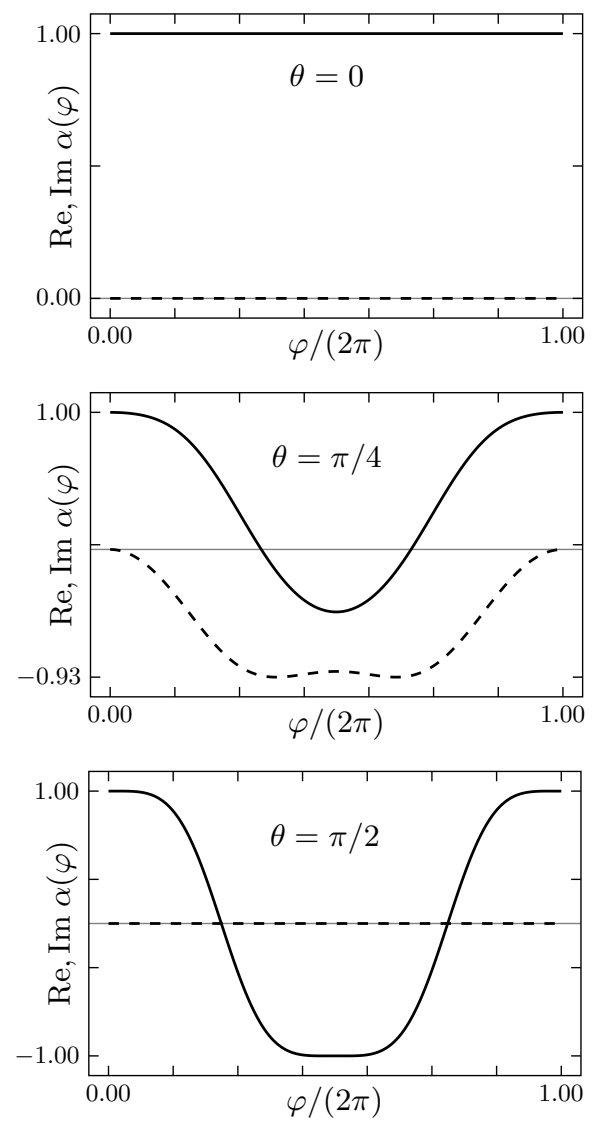

FIG. 4. Angular dependence of the eigenfunctions $\alpha$ of $A_{V_{\uparrow \downarrow}}$ (real parts solid, imaginary parts dashed) for $k_{F} \lambda=1$. Since the dipole orientation is in the $x z$-plane, we always have $\alpha(\varphi)=\alpha(-\varphi)$.

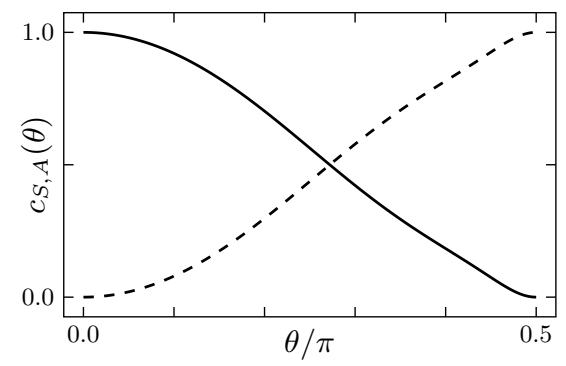

FIG. 5. The coefficients $c_{S}$ (solid) and $c_{A}$ (dashed) giving the symmetric (singlet) and antisymmetric (triplet) components of the interlayer pair wave function respectively, as a function of $\theta$ for $k_{F} \lambda=1$.

symmetric part writing

$$
\alpha(\varphi)=\underbrace{\frac{\alpha(\varphi)+\alpha(\varphi+\pi)}{2}}_{=: \alpha_{S}(\varphi)}+\underbrace{\frac{\alpha(\varphi)-\alpha(\varphi+\pi)}{2}}_{=: \alpha_{A}(\varphi)} .
$$

From this, we can define the symmetry coefficients

$$
c_{S, A}:=\frac{\int_{0}^{2 \pi}\left|\alpha_{S, A}(\varphi)\right|^{2} \mathrm{~d} \varphi}{\int_{0}^{2 \pi}|\alpha(\varphi)|^{2} \mathrm{~d} \varphi},
$$

where $c_{S}$ gives the singlet component of the pair wave function and $c_{A}$ the triplet component. Note that we have $c_{S}+c_{A}=1$ by construction.

In Fig. 5, we plot these symmetry coefficients for the interlayer pairing as a function of $\theta$ for the layer distance $k_{F} \lambda=1$. For $\theta=0$ where the interaction is inversion symmetric, $c_{S}=1$ and $c_{A}=0$ since the pair wave function is purely in the singlet channel. Both $c_{S}$ and $c_{A}$ are non-zero for $0<\theta<\pi / 2$ reflecting the mixed parity pairing. The triplet component $c_{A}$ increases with increasing $\theta$ whereas $c_{S}$ decreases, and the pair wave function is purely in the triplet channel with $c_{S}=0$ and $c_{A}=1$ for $\theta=\pi / 2$, where inversion symmetry is recovered.

\section{DISCUSSION}

Dipole-dipole interactions have been shown within Hartree-Fock theory to give rise to a elliptic Fermi surface for $\theta>0$ [26], and we should in principle include this in our analysis [27]. However, since the symmetry of the interaction is independent of such effects, the main results of this paper, the mixed parity interlayer pairing for $0<\theta<\pi / 2$ remain valid when Hartree-Fock effects are included. In particular, there will only be small quantitive changes to the phase diagram, especially for weak coupling.

Although our results were derived using a weak coupling approach, we expect them to hold also for stronger coupling, since they follow directly from the symmetry of the interaction. As the superfluid critical temperature increases with the coupling strength, this opens 
up the possibility to experimentally unambiguously observe mixed parity pairing with dipolar gases. Impressive experimental progress in trapping and cooling fermionic molecules with a large dipole moment have recently been reported $[28,29]$. The symmetry of the pair wave function can be detected in time-of-flight (TOF) experiments, as has been explicitly demonstrated for the intralayer triplet pairing [20]. TOF experiments have been successfully used to detect various correlation functions in atomic gases [30-32].

The interaction cannot be made too strong however, since the system is then predicted to enter a striped (charge-density-wave) phase [33-40]. We expect the stripes to suppress the $s$-wave pairing. On the other hand, $p$-wave pairing can co-exist with the striped phase, since it is mainly formed in regions left ungapped by the stripes [20]. This leads to the intriguing possibility of forming a supersolid with interlayer pairing and intralayer stripes.

Mixed parity pairing is predicted to lead to several intriguing effects related to transport and topological properties of noncentrosymmetric crystals [41-43]. It is an interesting question whether similar effects appear for the present system, where the lack of inversion symmetry appears through the interaction and not through an underlying crystal structure.

\section{CONCLUSIONS}

We demonstrated that dipolar fermions residing in two parallel layers can form interlayer pairing with both singlet and triplet components. This mixed parity pairing arises because the interlayer interaction is not inversion symmetric when the dipole moments are tilted with respect to the normal of the plane. We used an efficient and accurate eigenvalue method to investigate the competition between the interlayer pairing and the intralayer pairing, which is in the triplet channel, and the result- ing zero temperature phase diagram was calculated. We showed how the interlayer pair wave function smoothly changes from singlet symmetry for $\theta=0$ to triplet symmetry for $\theta=\pi / 2$. Our results indicate that dipolar gases can be used to unambiguously observe mixed parity pairing.

\section{Appendix A: Numerical Method}

In order to examine the spectrum of the integral operator (6), we use the linear span of the $N \gg 1$ characteristic functions

$$
b_{n}(\mathbf{p})=\frac{1}{\mathcal{N}_{n}} \chi_{\left[\frac{2 \pi}{N}(n-1), \frac{2 \pi}{N} n\right]}\left(\varphi_{\mathbf{p}}\right)
$$

where $n=1, \ldots, N$. Here, $\varphi_{\mathbf{p}}$ denotes the polar angle corresponding to $\mathbf{p}$. With the normalization constants

$$
\mathcal{N}_{n}^{2}=\sqrt{\frac{2}{a^{2}+b^{2}}} \int_{\frac{2 \pi}{N}(n-1)}^{\frac{2 \pi}{N} n} \mathrm{~d} \varphi \sqrt{1+\frac{a^{2}-b^{2}}{a^{2}+b^{2}} \cos (2 \varphi)},
$$

these functions $\left(b_{n}\right)_{n=1}^{N}$ form an orthonormal basis with regard to the inner product

$$
\langle f \mid g\rangle:=\frac{2}{a^{2}+b^{2}} \int_{\Omega_{a, b}} \overline{f(\mathbf{p})} g(\mathbf{p}) \mathrm{d} p,
$$

where $\Omega_{a, b}$ denotes the ellipse with half-axes $a$ and $b$. The eigenvalues of the matrix formed by $M_{m n}=\left\langle b_{m} \mid A_{V} b_{n}\right\rangle$ are an approximation for the desired spectrum. Note that $M$ is in fact hermitian. In the calcuations, we used $N=600$ functions.

\section{ACKNOWLEDGMENTS}

Partial financial support from the DFG grant GRK 1838 is gratefully acknowledged. GMB would like to acknowledge the support of the Villum Foundation via grant VKR023163 and ESF POLATOM network.
[1] P. Anderson, Journal of Physics and Chemistry of Solids 11, 26 (1959).

[2] P. W. Anderson, Phys. Rev. B 30, 4000 (1984).

[3] L. P. Gor'kov and E. I. Rashba, Phys. Rev. Lett. 87, 037004 (2001).

[4] N. Hayashi, K. Wakabayashi, P. A. Frigeri, and M. Sigrist, Phys. Rev. B 73, 092508 (2006).

[5] M. Nishiyama, Y. Inada, and G.-q. Zheng, Phys. Rev. Lett. 98, 047002 (2007).

[6] H. Q. Yuan, D. F. Agterberg, N. Hayashi, P. Badica, D. Vandervelde, K. Togano, M. Sigrist, and M. B. Salamon, Phys. Rev. Lett. 97, 017006 (2006).

[7] I. Bonalde, W. Brämer-Escamilla, and E. Bauer, Phys. Rev. Lett. 94, 207002 (2005).
[8] K. Izawa, Y. Kasahara, Y. Matsuda, K. Behnia, T. Yasuda, R. Settai, and Y. Onuki, Phys. Rev. Lett. 94, 197002 (2005).

[9] H. Mukuda, S. Nishide, A. Harada, K. Iwasaki, M. Yogi, M. Yashima, Y. Kitaoka, M. Tsujino, T. Takeuchi, R. Settai, Y. Ōnuki, E. Bauer, K. M. Itoh, and E. E. Haller, Journal of the Physical Society of Japan 78, 014705 (2009), http://dx.doi.org/10.1143/JPSJ.78.014705.

[10] U. R. Fischer, Phys. Rev. A 73, 031602 (2006).

[11] Q. Li, E. H. Hwang, and S. Das Sarma, Phys. Rev. B 82, 235126 (2010).

[12] A. Pikovski, M. Klawunn, G. V. Shlyapnikov, and L. Santos, Phys. Rev. Lett. 105, 215302 (2010). 
[13] N. T. Zinner, B. Wunsch, D. Pekker, and D.-W. Wang, Phys. Rev. A 85, 013603 (2012).

[14] M. A. Baranov, A. Micheli, S. Ronen, and P. Zoller, Phys. Rev. A 83, 043602 (2011).

[15] A. Camacho-Guardian, G. A. Domínguez-Castro, and R. Paredes, ArXiv e-prints (2015), arXiv:1511.06311 [cond-mat.quant-gas].

[16] N. Matveeva and S. Giorgini, Phys. Rev. A 90, 053620 (2014).

[17] M. Sigrist, in American Institute of Physics Conference Series, American Institute of Physics Conference Series, Vol. 1162, edited by A. Avella and F. Mancini (2009) pp. $55-96$.

[18] G. M. Bruun and E. Taylor, Phys. Rev. Lett. 101, 245301 (2008).

[19] L. M. Sieberer and M. A. Baranov, Phys. Rev. A 84, 063633 (2011).

[20] Z. Wu, J. K. Block, and G. M. Bruun, Phys. Rev. B 91, 224504 (2015).

[21] C. Hainzl, E. Hamza, R. Seiringer, and J. P. Solovej, Comm. Math. Phys. 281, 349 (2008).

[22] R. L. Frank, C. Hainzl, S. Naboko, and R. Seiringer, J. Geom. Anal. 17, 559 (2007).

[23] C. Hainzl and R. Seiringer, Phys. Rev. B 77, 184517 (2008).

[24] C. Hainzl and R. Seiringer, Math. Nachr. 283, 489 (2010).

[25] C. Hainzl and R. Seiringer, (2015), arXiv:1511.01995v1.

[26] T. Miyakawa, T. Sogo, and H. Pu, Phys. Rev. A 77, 061603 (2008).

[27] G. Bräunlich, C. Hainzl, and R. Seiringer, Reviews in Mathematical Physics 26, 1450012 (2014), http://www.worldscientific.com/doi/pdf/10.1142/S0129055X14500123.

[28] M.-S. Heo, T. T. Wang, C. A. Christensen, T. M. Rvachov, D. A. Cotta, J.-H. Choi, Y.-R. Lee, and W. Ket- terle, Phys. Rev. A 86, 021602 (2012).

[29] J. W. Park, S. A. Will, and M. W. Zwierlein, Phys. Rev. Lett. 114, 205302 (2015).

[30] M. Greiner, C. A. Regal, J. T. Stewart, and D. S. Jin, Phys. Rev. Lett. 94, 110401 (2005).

[31] S. Folling, F. Gerbier, A. Widera, O. Mandel, T. Gericke, and I. Bloch, Nature 434, 481 (2005).

[32] T. Rom, T. Best, D. van Oosten, U. Schneider, S. Folling, B. Paredes, and I. Bloch, Nature 444, 733 (2006).

[33] Y. Yamaguchi, T. Sogo, T. Ito, and T. Miyakawa, Phys. Rev. A 82, 013643 (2010).

[34] M. Babadi and E. Demler, Phys. Rev. B 84, 235124 (2011).

[35] J. K. Block, N. T. Zinner, and G. M. Bruun, New Journal of Physics 14, 105006 (2012).

[36] M. M. Parish and F. M. Marchetti, Phys. Rev. Lett. 108, 145304 (2012).

[37] B. P. van Zyl, W. Kirkby, and W. Ferguson, Phys. Rev. A 92, 023614 (2015).

[38] J. K. Block and G. M. Bruun, Phys. Rev. B 90, 155102 (2014).

[39] A. Keles and E. Zhao, ArXiv e-prints (2015), arXiv:1512.06147 [cond-mat.quant-gas].

[40] Z. Wu, J. K. Block, and G. M. Bruun, Scientific Reports 6, $19038 \mathrm{EP}$ (2016).

[41] Y. Tanaka, T. Yokoyama, A. V. Balatsky, and N. Nagaosa, Phys. Rev. B 79, 060505 (2009).

[42] M. Sato and S. Fujimoto, Phys. Rev. B 79, 094504 (2009).

[43] Y. Tada, N. Kawakami, and S. Fujimoto, New Journal of Physics 11, 055070 (2009), arXiv:0902.3043 [condmat.supr-con]. 